EVS26

Los Angeles, California, May 6-9, 2012

\title{
Towards onboard Li-ion battery state-of-health diagnosis by a virtual sensor
}

\author{
Rémy MINGANT, Julien BERNARD, Valérie SAUVANT-MOYNOT \\ IFP Energies nouvelles, Rond-point de l'échangeur de Solaize, BP 3, 69360 Solaize, France \\ remy.mingant@ifp.fr
}

\begin{abstract}
The development of improved State-of-Health $(\mathrm{SoH})$ diagnosis methods is a current research topic for battery-powered applications. For instance, Hybrid Electric Vehicles (HEV) are in rapid extension and create a strong demand for an accurate and reliable $\mathrm{SoH}$ indicator during operation. Such an indicator is a key parameter required to optimize battery energy management and to track the degradation of the system performances. Electrochemical impedance spectroscopy is one of the most promising methods for characterizing ageing effects of secondary batteries online because it can be sensitive to different ageing mechanisms.

This paper presents a method to measure the electrochemical transfer function of the cell at different frequencies simultaneously with the data treatment of free current and voltage during operation. Impedance parameters were then estimated by a non linear least square algorithm. Based on this approach, an experimental investigation of ageing effects of a lithium ion battery was carried out. After $1400 \mathrm{HEV}$ cycles, the battery showed a $7 \%$ decreased capacity, and $8 \%$ increased resistance at $0.5 \mathrm{~Hz}$. Modelling results show that charge transfer resistance have a similar evolution in function of ageing time than the resistance of battery measured by electrochemical impedance spectroscopy. To our knowledge on the topic, this constitutes a substantial step towards SoH indicator that could be reliable and precise enough under realistic user conditions.
\end{abstract}

Keywords: Li-ion, State of Health, Impedance spectroscopy, ageing

\section{Introduction}

The development of methods for the determination of state of charge and state of health of batteries is a key research topic in the field of energy storage systems.
Electrified vehicles (HEV, plug-in hybrid and electric) are in fast evolution, creating a strong demand of safe and accurate indicators for the state-of-charge (SoC) and state-of-health of batteries. Such indicators are essential to optimize energy management and avoid overcharging; this development is thus a necessary step to enhance the lifespan of the battery. Indeed, a long life cycle 
reduces the cost of energy storage, and improves the reliability and security of the system. The purpose of this study is to establish a status indicator of ageing of the battery sufficiently accurate for the intended applications.

This paper presents a method to determine the electrical impedance of an electrochemical system by processing the signal voltage $\mathrm{U}$ and current I from a Li-ion battery in conventional HEVs use.

\section{Li-ion cells ageing factors}

Several experimental technics for the estimation of the batteries state are referenced in the literature and patents. Among them, electrochemical impedance spectroscopy (EIS) is widely investigated because few intrusive [1].

There are several methods for determining the parameters of battery by EIS:

- An approach based on the measurement of internal resistance, which is commonly dependent on the SoC and SoH of the battery [2]. In this case, the classical resistance is measured at a frequency of $1 \mathrm{kHz}$ or higher. On this basis, smart sensors have been developed to determine the state of lead-acid batteries by applying pulses of current [3].

- An alternative method is to measure the resistance when the imaginary part of the impedance is equal to 0 . This characteristic frequency enables to estimate the battery state of charge for Ni-MH and Ni-Cd batteries [1, 3-5].

- Another approach is based on the adjustment of impedance spectra by an equivalent electrical circuit, where some values of electrical parameters $(\mathrm{R}, \mathrm{C})$ depend on the $\mathrm{SoC}$ and $\mathrm{SoH}$ of batteries [6-8]. A method developed in part by IFPEN was based on a multifactorial adjustment of these resistance and capacity values to obtain an accurate estimation of the internal characteristics of a battery [9]. In addition, the literature also presents various methods for determining the impedance of the batteries [1,10]. IFPEN recently proposed an innovative method by determining so called "electrochemical impedance like" of Li-ion batteries by using the free signals $[\mathrm{U}, \mathrm{I}]=\mathrm{f}(\mathrm{t})$ of a battery during operation and calculating the transfer function from it $[11,12]$.

\section{3 "EIS-like" measurement by signal treatment}

The power spectral density (PSD) is a mathematical function used to represent the different spectral components of a signal. It is equal to the square of the modulus of the Fourier transform of $X(t)$, denoted $X(f)$ reported in half the acquisition time (1):

$$
\Psi_{x}(f)=\frac{2}{T}|X(f)|^{2}
$$

where $\mathrm{T}$ is the integration time.

There are also cross-power spectral densities which are composed of a combination of two Fourier transform X (f) and Y (f) (2):

$$
\Psi_{x y}(f)=\frac{2}{T} X(f) Y^{*}(f)
$$

The impedance $\mathrm{Z}$ is classicaly calculated using the formula (3):

$$
Z(f)=\frac{V(f)}{I(f)}=\frac{\frac{2}{T} V(f) I^{*}(f)}{\frac{2}{T} I(f) I^{*}(f)}=\frac{\Psi_{I V}(f)}{\Psi_{I}(f)}
$$

However, this formula does not practically provide a good accuracy of the impedance. It is therefore necessary to divide the signal into $\mathrm{N}$ blocks, then to take the average before calculating the impedance (4).

$$
Z(f)=\frac{\frac{1}{N} \sum_{j=1}^{N} \frac{2}{T} V_{j}(f) I_{j}^{*}(f)}{\frac{1}{N} \sum_{j=1}^{N} \frac{2}{T} I_{j}(f) I_{j}^{*}(f)}=\frac{\frac{1}{N} \sum_{j=1}^{N} \Psi_{U I}(f)}{\frac{1}{N} \sum_{j=1}^{N} \Psi_{I}(f)}
$$

This division of blocks causes a reduction of studied frequencies, because of the decrease in the amount of information processed at any time. Thus, for a sample of $\mathrm{n}$ values, measured at constant time step, $f$, the frequency is a vector comprising the numbers from 1 to $\mathrm{n}$, divided by the duration of the test. Thus, a reduction in sample size also results in the number of frequencies, and the number of low frequencies.

In addition, according to the Nyquist-Shannon theorem:

"If a function $\mathrm{x}(\mathrm{t})$ contains no frequencies higher than B Hertz, it is completely determined by giving its ordinates at a series of points spaced 1/(2B) seconds apart." 
Thus, higher frequencies must be removed (which reduces even more the number of frequencies of study).

There is also a source of uncertainty about the signal. Indeed, it is not white noise, and thus the power spectral density is not constant with frequency, implying ratio U (f) / I (f) which can be quite random (Figure 1a). Thus, a filter system of the power spectral density has been developed to select only the frequency with the most important power spectral densities (Figure 1b, the frequencies of which the DSP is greater than I (DSPI max) / 10).
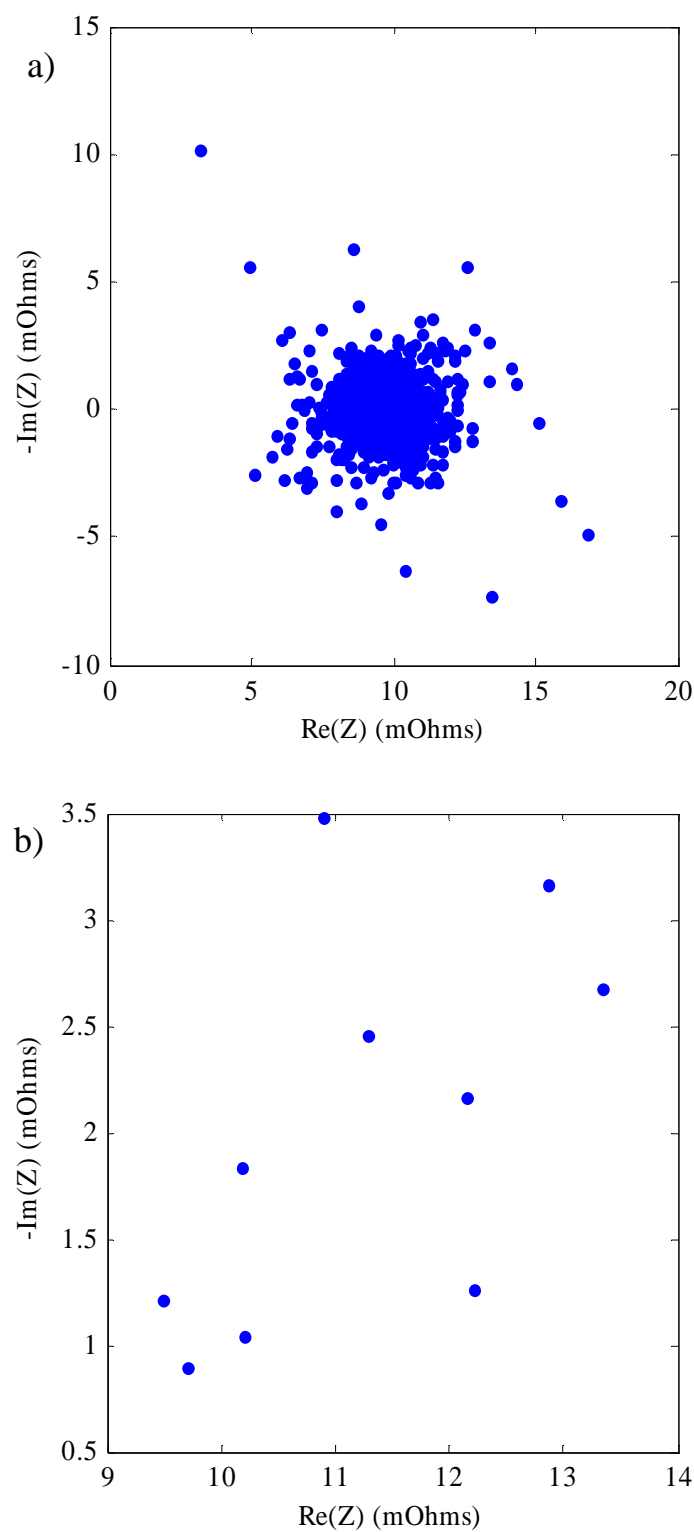

Figure 1: Impedance spectrum of an A123 Systems 2.3 Ah cell from an HEV profile without DSP filter (a), and with DSP filter (b)
In order to increase the relative accuracy of the electrochemical impedance it was then proposed to cut the signal, in order to reduce the number and frequency range studied.

In order to obtain an impedance with good accuracy over a large part of the frequency range, one solution is to treat the same signal several times, but with a "cutting" becoming weaker (Figure 2). Example for the signal of 80000 values studied previously: the first cutting of 5000 sections of 16 values and 2500 sections of 32 values ... $80000 / \mathrm{n}$ sections of $\mathrm{n}$ values, where $\mathrm{n}$ is a nonzero integer. It is worth noting that this type of treatment does not allow to obtain impedance spectra on the same frequency range as a standard measurement made from sinusoids (Figure 2).

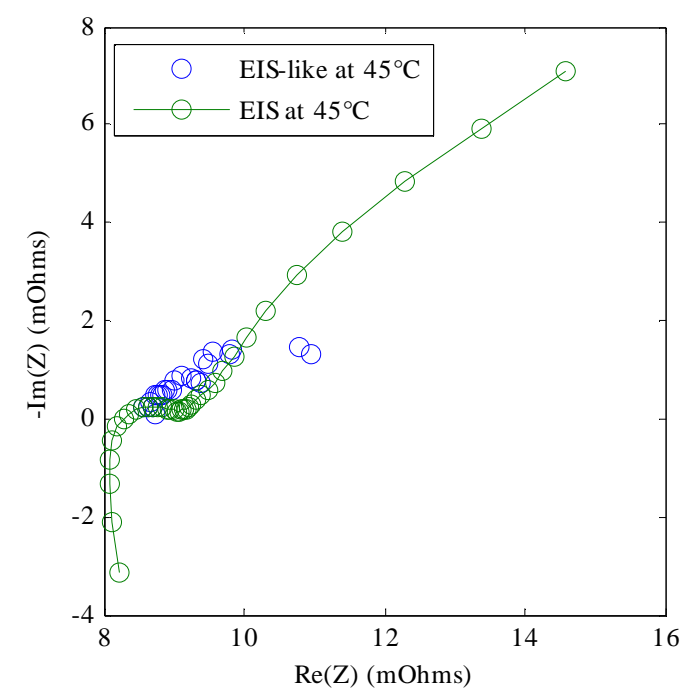

Figure 2: Impedance spectra of an A123 Systems 2.3 Ah obtained from the method of "successive cutting" from a signal $[\mathrm{U}, \mathrm{I}]=\mathrm{f}(\mathrm{t})$ road (frequencies of 0.78 to 0.015

$\mathrm{Hz}$ ), compared with an impedance measured on an identical cell at the same temperature (frequencies from 10000 to $0.005 \mathrm{~Hz})$. 


\section{Hybrid vehicle ageing protocol (HEV)}

Commercial batteries (2.3 Ah A123 Systems cylindrical LFP/C cell) were selected to test the diagnosis method. The nominal voltage is $3.3 \mathrm{~V}$ and voltage limits are $2 \mathrm{~V}$ and $3.6 \mathrm{~V}$.

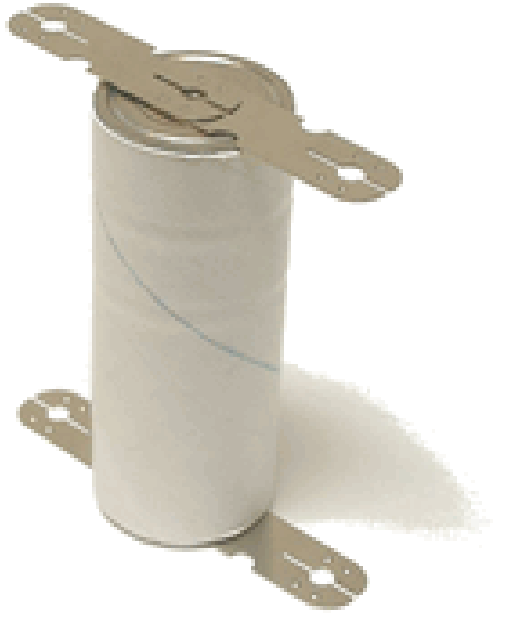

Figure 3: Studied A123 Systems cell

In order to study the capability of "EISlike" measurements as a diagnosis tool for $\mathrm{SoH}$ of Li-ion cells, a test procedure including ageing phases has been defined to characterise Li-ion cells before and along ageing.

Ageing phases were defined by $\mathrm{HEV}$ current profiles $(15 \mathrm{~min})$ followed by rest period (1h15 min), in order to mimic real operating condition (Figure 4).

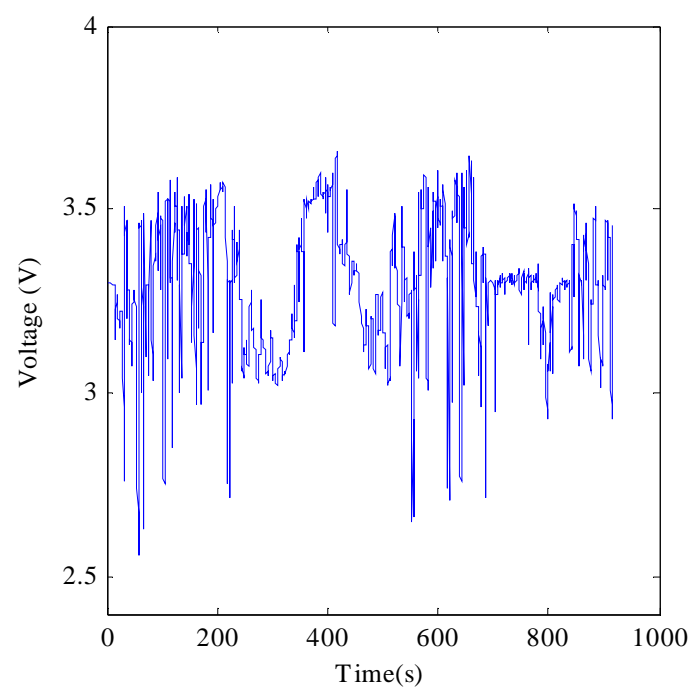

Figure 4: Voltage of an A123 Systems 2.3 Ah cell submitted to HEV current profile.

\section{First results}

An "EIS-like" spectrum resulting from HEV signal is presented on Figure 5 as an example. These Bode plots show noisy curves, however, comparison with others spectra collected by a conventional EIS-measuring tool can be made.
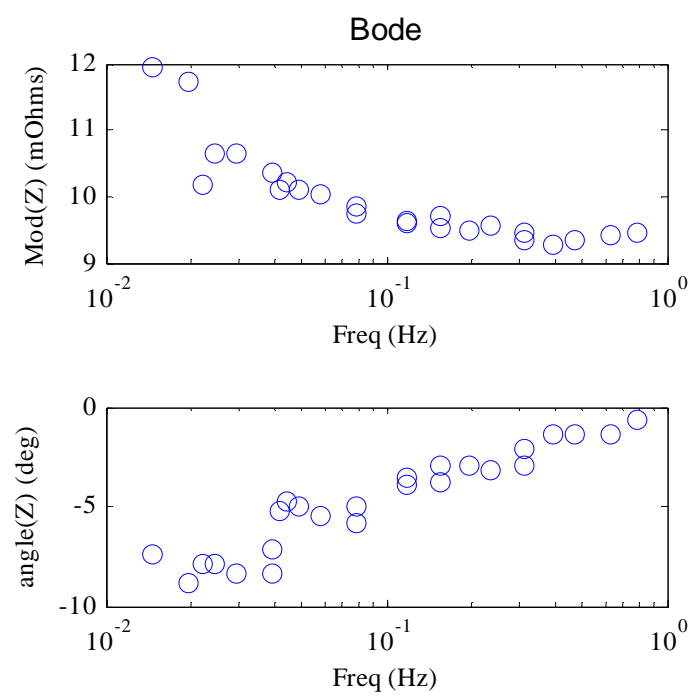

Figure 5: Bode diagram of the "EIS-like" calculated by means of Fourier transform.

The Figure 6 shows the Nyquist plots for A123 Systems battery before and after given ageing cycles. The diagrams are composed of two halfcircle-like loop. As one can observe, while the shape does not change much with ageing, the whole impedance diagram shifts towards upper real values when the number of cycles increases. 


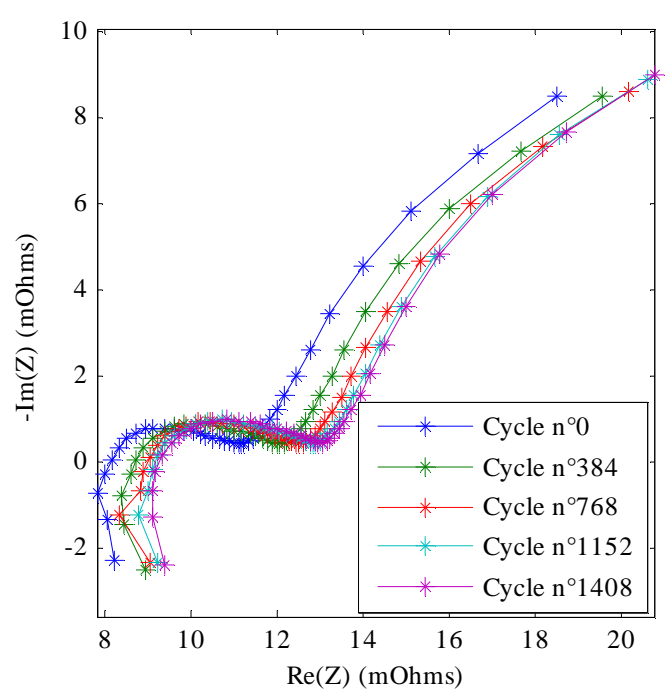

Figure 6: Comparison between the impedance spectra of an A123 Systems cell before and after several ageing periods (Nyquist representation).

The evolution of the "EIS-like" diagrams of Liion cells measured by Fourier transformation are given in Figure 7 as a function of ageing. In this figure, each impedance curve has almost the same shape. once again,a shift between each curve can be observed towards higher real values as studied from Figure 6.

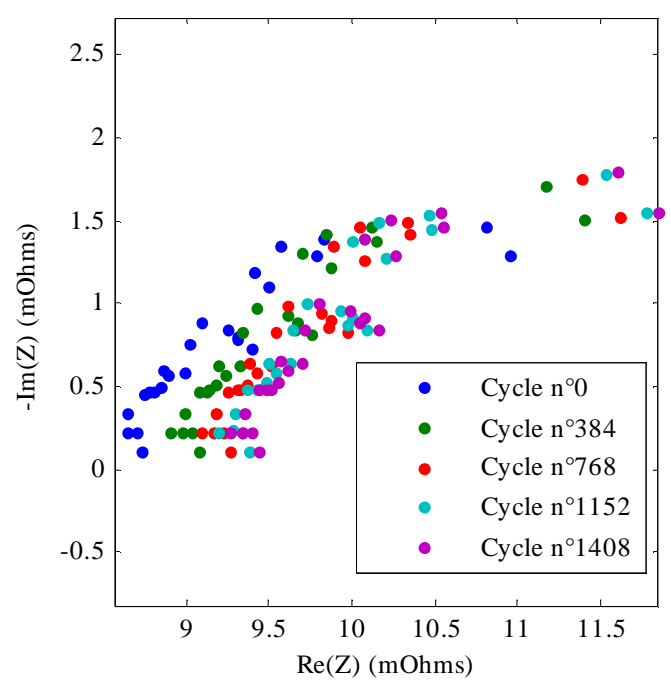

Figure 7: Comparison between the impedance-like spectra of an A123 Systems cell before and after several ageing periods (Nyquist representation).

Equivalent circuit used for impedance spectra fitting is presented on Figure 8. R0 represents the ohmic resistance at high frequencies while the electrochemical processes are in a first approximation modelled by a capacitor $(\mathrm{C} 1)$ in parallel with the charge transfer resistance $\mathrm{R} 1$.

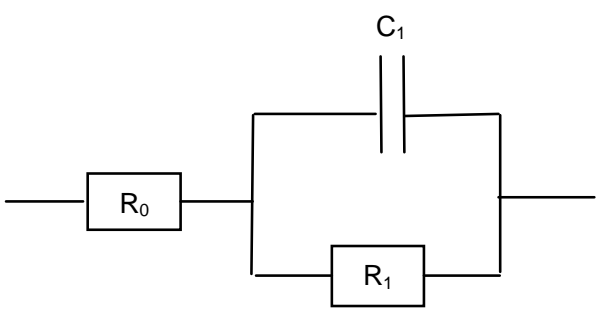

Figure 8: Simple electrical equivalent circuit used to fit the A123 Systems cell EIS diagrams

The parameters of the equivalent circuit were estimated by numerical optimization (Figure 9). For this purpose a nonlinear curve-fitting optimization based on the least-square method was used for each impedance spectrum. The results show that parameters like $\mathrm{R}, \mathrm{C}$ can vary during ageing [8].

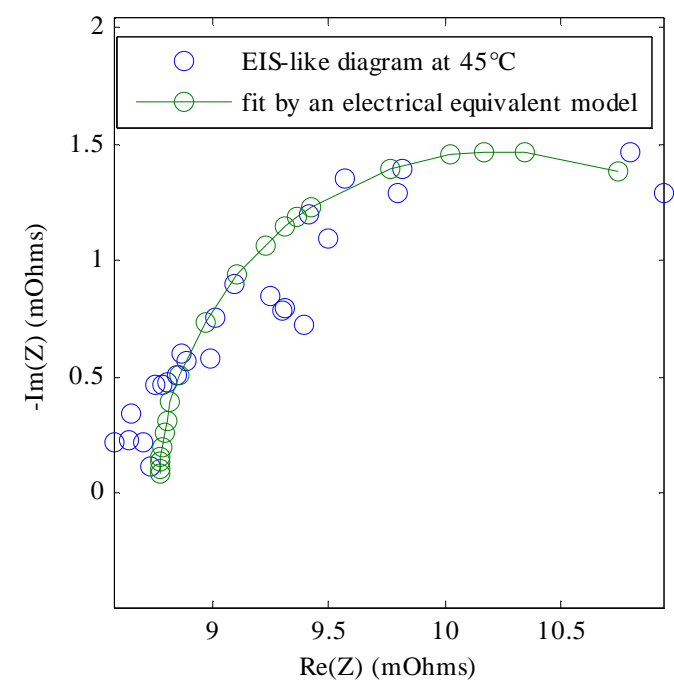

Figure 9: Fit of the impedance spectra of an A123 Systems 2.3 Ah cell with a simple equivalent circuit (no ageing)

The Figure 10 represents the resistances calculated by the "EIS-like" method as a function of HEV cycle number (Figure 10 a) in comparison with resistances taken at $0.5 \mathrm{~Hz}$ with a conventional EIS measurement at $23{ }^{\circ} \mathrm{C}$ (Figure 10 b). Both graphs show similar trends as a function of cycle number. 

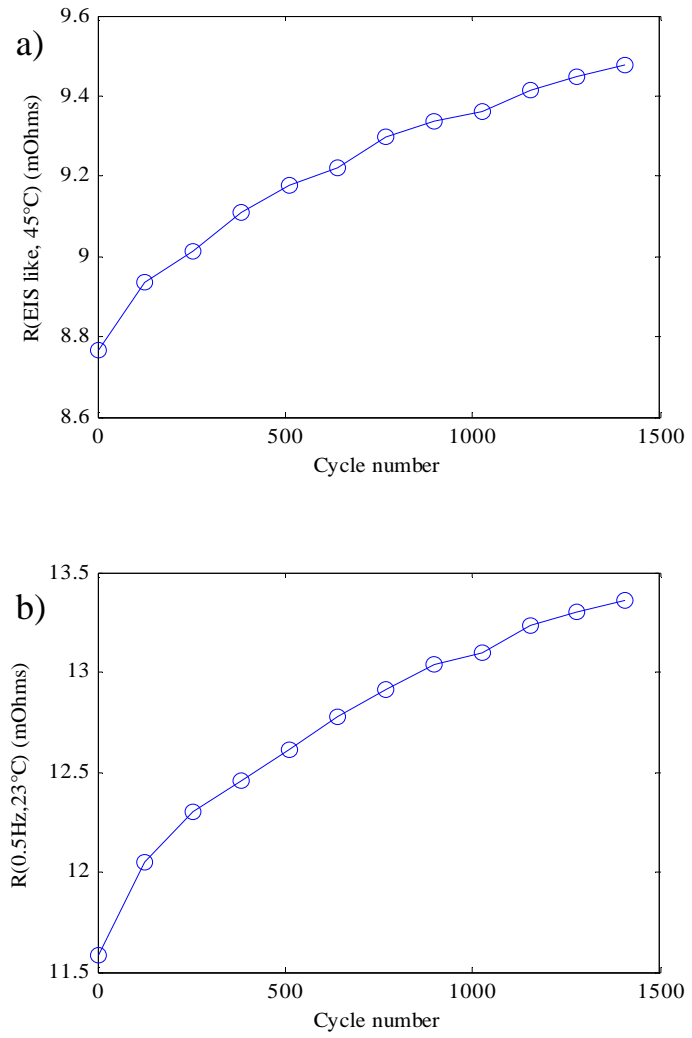

Figure 10: Evolution of cell's resistance as a function of cycle number, a) calculated by EIS like method (45 $\left.{ }^{\circ} \mathrm{C}\right)$, b) measured by conventional EIS method at 0.5 $\mathrm{Hz}, 23{ }^{\circ} \mathrm{C}$.

\section{Conclusions:}

A novel method was proposed to determine the impedance diagrams of a Li-ion cell based on the electrochemical transfer function calculated from the input I/output $\mathrm{U}$ signal during use. In order to compare each impedance collected during ageing, these diagrams were fitted according to a simple electrical equivalent circuit model. The evolution of the parameters as a function of ageing was studied revealing a monotonous change of the resistance with the ageing of the battery. Thus, the prediction of the SoH could be obtained by analyzing the model parameters of the fitted "EIS-like" diagrams. Therefore, this work could serve to support the promotion of new non-intrusive diagnosis sensors of Li-ion batteries.

\section{References}

[1] F. Huet, J.Power Sources 70 (1998) 59-69

[2] S. Klutz and B. Faust, US patent, 6191590 (2001)

[3] M. Coleman, C.K. Lee, W.G. Hurley, C. Zhu, US patent, 7692410 (2010)

[4] R.W. De Doncker, A. Hammouche, E. Karden, B. Fricke. US Patent, 200790843 (2007)

[5] A. Hammouche, E. Karden, R.W. De Doncker, J Power Sources, 127 (2004) 105

[6] J. Tinnemeyer, US patent, 6778913 (2002)

[7] K. Champlin, US patent, 6037777 (1998)

[8] U. Troltzsch, O. Kanoun, H.R. Trankler, Electrochim. Acta 51 (2006) 1664

[9] R. Mingant et al, FR patent, 2956486 (2010)

[10] C. Gabrielli, F. Huet, M. Keddam, J.Electroanal.Chem. 335 (1992) 33-53

[11] R. Mingant, J. Bernard and V. SauvantMoynot, FR Patent 2956743 (2011)

[12] R. Mingant, J. Bernard and V. SauvantMoynot, A. Delaille, S. Mailley, J-L. Hognon, F. Huet, ECS Transactions 33 (2011) 41-53

\section{Authors}

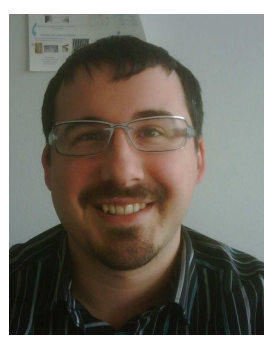

\author{
Rémy Mingant, Ph.D \\ Research Engineer \\ IFP Energies nouvelles \\ BP3 - 69360 Solaize - France \\ Tel: 33437702261 \\ Fax: 33478022141 \\ remy.mingant@ifpen.fr
}

R. Mingant joined IFP Energies nouvelles in 2009 with a $\mathrm{PhD}$ from Grenoble Electrochemistry National School (ENSEEG). $\mathrm{He}$ is in charge of experimental battery testing, data processing and modeling in the Electrochemical and Materials Department.

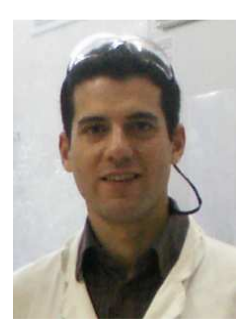

Julien Bernard, Ph.D. Battery project leader IFP Energies nouvelles BP3 - 69360 Solaize - France

Tel: 33437702223

Fax: 33437702141

julien.bernard@ifpen.fr 
J. Bernard is in charge of $R \& D$ on electrochemical storage systems in the Electrochemical \& Materials Department since 2007. He joined IFP Energies nouvelles with an Engineering degree of the Grenoble Electrochemistry National School (ENSEEG) and $\mathrm{PhD}$ in electrochemistry.

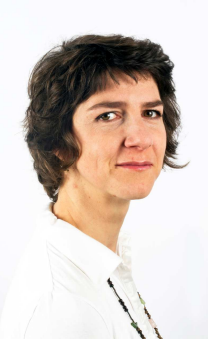

Valérie Sauvant-Moynot, Ph.D

Head of the electrochemical and materials department

IFP Energies nouvelles, Ets de Lyon

BP3 - 69360 Solaize - France

Tel: 33478022685

Fax: 33478022141

valerie.sauvant@ifpen.fr

Joined the Materials Department of IFP Energies nouvelles in 1999 with an Engineering degree of the Chemical National School of Paris (ENSCP) and a PhD in Polymer Science. Since 2006 leads the development of the "battery" activity at IFP Energies nouvelles as project manager for the Transport Business Unit. Became head of of the electrochemical and materials department in 2010. 